\title{
Repeated Interaction Quantum Systems
}

\author{
Marco Merkli*† \\ Department of Mathematics and Statistics \\ Memorial University of Newfoundland \\ St. John's, NL \\ Canada A1C 5S7
}

December 10, 2007

\begin{abstract}
We consider a quantum system interacting sequentially with elements of a chain of independent quantum subsystems. We treat two kinds of such repeated interaction systems: deterministic and random ones. In both cases we show that, under suitable conditions, the system approaches an asymptotic state in the large time limit, and we construct that state.

Our methods are based on the analysis of products of operators generating the dynamics at each step in the process of repeated interaction. In the random case, we obtain results about infinite products of independent, identically distributed random matrices.
\end{abstract}

\section{Introduction}

Consider a quantum system $\mathcal{S}$ which interacts with another one, $\mathcal{E}_{1}$, during a time interval $\left[0, \tau_{1}\right)$, then for times $\left[\tau_{1}, \tau_{1}+\tau_{2}\right), \mathcal{S}$ interacts with another system $\mathcal{E}_{2}$, and so on. The assembly of the $\mathcal{E}_{k}$, which we suppose to be independent of each other (i.e., not directly coupled), is called a chain, $\mathcal{C}=\mathcal{E}_{1}+\mathcal{E}_{2}+\cdots$. The system $\mathcal{S}+\mathcal{C}$ is called a repeated interaction quantum system. One may think of $\mathcal{S}$ as being the system of interest, say a particle enclosed in a container, and of $\mathcal{C}$ as a chain of measuring apparatuses $\mathcal{E}_{k}$ that are brought into contact with the particle in a sequential manner. The system $\mathcal{S}$ is an open quantum system, coupled to the "environment" $\mathcal{C}$. Our goal to study the influence of $\mathcal{C}$ on $\mathcal{S}$, and to describe the (asymptotic) dynamics of the latter system.

The theoretical and practical importance of repeated interaction quantum systems is exemplified by systems of radiation-matter coupling, where atoms interact with modes of the quantized electromagnetic field. In this setting, the system $\mathcal{S}$ describes one or

\footnotetext{
${ }^{*}$ This paper is based on a talk presented at the ICMP 2006 in Rio de Janero. All the results presented here have been obtained in collaboration with with Laurent Bruneau and Alain Joye. See also $[4,5,6]$

${ }^{\dagger}$ Email: merkli@math.mun.ca, http://www.math.mun.ca/〜merkli/
} 


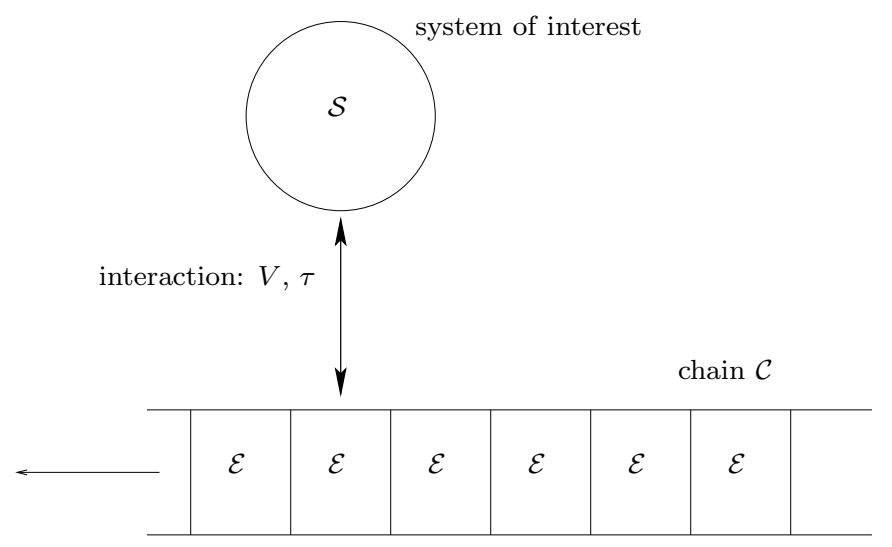

Figure 1: A repeated interaction system

several modes of the field in a cavity and the chain $\mathcal{C}$ represents a beam of atoms $\mathcal{E}$ injected into the cavity. So-called "One-Atom Masers", where the beam in tuned in such a way that at each given moment a single atom is inside a microwave cavity have been experimentally realized in laboratories $[9,11]$. After interaction, the atoms encode certain properties of the field that can be measured after they exit the cavity.

We distinguish two classes of models of repeated interaction quantum systems. In deterministic models, each system $\mathcal{E}_{k}$ is the a copy of a single quantum system $\mathcal{E}$, and the interactions between $\mathcal{S}$ and $\mathcal{C}$ are determined by a fixed interaction time $\tau>0$ and interaction operator $V$ (acting on $\mathcal{S}$ and $\mathcal{E}$ ), $\tau, V$ being independent of the interaction step. While deterministic models are interesting quantum dynamical systems in their own right, it is clear that they are idealized mathematical models, if supposed to describe physical experiments (as for instance the "One-Atom Maser"). Indeed, in actual experiments, neither the way of interaction, nor the interaction time, nor the elements $\mathcal{E}$ will be exactly the same for each step in the process. Rather, the interaction time should be considered to be random, for instance given by a Gaussian (or a uniform) distribution around a mean value. Further, the atoms in experiments are ejected from an atom oven, then they are cooled down to a wanted temperature before entering the cavity. Of course one cannot have absolute control over their preparation or their interaction with the field in the cavity. Thus the state of the incoming atoms should also be taken random, for instance determined by a temperature that fluctuates slightly around a mean temperature. It is therefore important to develop a theory that allows for random repeated interaction systems, which is the second class of systems we consider. The randomness may have different sources, it may come from fluctuations in the incoming elements $\mathcal{E}_{k}$, or in the interaction, via random interaction times $\tau_{k}$ and/or random interaction operators $V$.

Literature. The reader will find in $[4,5,6]$ a more detailed list of related works. Mathematical work on systems similar to the ones considered here is done in [12], using entirely different methods. The part of our work dealing with products of random matrices and random ergodic theorems is linked to many other works, see the references in $[5]$. 
Acknowledgements. I am grateful to Bruno Nachtergaele for inviting me to give a talk at the Mathematical Physics section of the ICMP 2006. My thanks also go to the organizers of the congress, in particular to Vladas Sidoravicius, for impecable organization and for financial support. I thank Laurent Bruneau and Alain Joye for their friendship and collaboration, which has produced the results presented here.

\section{Deterministic systems}

It is more convenient for the reader to first consider deterministic models, and to pass to random ones in a second step. We describe our systems within the framework of algebraic quantum statistical mechanics, an introduction to which can be found e.g. in $[2,3]$.

\subsection{Mathematical description}

The determinisitc models consist of a system $\mathcal{S}$ which is coupled to a chain $\mathcal{C}=\mathcal{E}+\mathcal{E}+$ .. of identical elements $\mathcal{E}$. We describe $\mathcal{S}$ and $\mathcal{E}$ as $W^{*}$-dynamical systems $\left(\mathfrak{M}_{\mathcal{S}}, \alpha_{\mathcal{S}}^{t}\right)$ and $\left(\mathfrak{M}_{\mathcal{E}}, \alpha_{\mathcal{E}}^{t}\right)$, where $\mathfrak{M}_{\mathcal{S}}, \mathfrak{M}_{\mathcal{E}}$ are von Neumann algebras of "observables" acting on the Hilbert spaces $\mathcal{H}_{\mathcal{S}}, \mathcal{H}_{\mathcal{E}}$, respectively, and where $\alpha_{\mathcal{S}}^{t}$ and $\alpha_{\mathcal{E}}^{t}$ are ( $\sigma$-weakly continuous) groups of $*$ automorphisms describing the Heisenberg dynamics. In this paper, we consider the situation $\operatorname{dim} \mathcal{H}_{\mathcal{S}}<\infty$ and $\operatorname{dim} \mathcal{H}_{\mathcal{E}} \leq \infty$.

We assume that there are distinguished reference vectors $\psi_{\mathcal{S}} \in \mathcal{H}_{\mathcal{S}}$ and $\psi_{\mathcal{E}} \in \mathcal{H}_{\mathcal{E}}$, determining states on $\mathfrak{M}_{\mathcal{S}}$ and $\mathfrak{M}_{\mathcal{E}}$ which are invariant w.r.t. $\alpha_{\mathcal{S}}^{t}$ and $\alpha_{\mathcal{E}}^{t}$, respectively, and we assume that $\psi_{\mathcal{S}}$ and $\psi_{\mathcal{E}}$ are cyclic and separating for $\mathfrak{M}_{\mathcal{S}}$ and $\mathfrak{M}_{\mathcal{E}}$, respectively. One may take equilibrium (KMS) vectors for these reference vectors.

The Hilbert space of the chain $\mathcal{C}$ is defined to be the infinite tensor product

$$
\mathcal{H}_{\mathcal{C}}=\otimes_{m \geq 1} \mathcal{H}_{\mathcal{E}}
$$

w.r.t. the reference vector ${ }^{1}$

$$
\psi_{\mathcal{C}}=\psi_{\mathcal{E}} \otimes \psi_{\mathcal{E}} \cdots
$$

We introduce the von Neumann algebra

$$
\mathfrak{M}_{\mathcal{C}}=\otimes_{m \geq 1} \mathfrak{M}_{\mathcal{E}}
$$

acting on $\otimes_{m \geq 1} \mathcal{H}_{\mathcal{E}}$, which is obtained by taking the weak closure of finite linear combinations of operators $\otimes_{m \geq 1} A_{m}$, where $A_{m} \in \mathfrak{M}_{\mathcal{E}}$ and $A_{m}=\mathbb{1}_{\mathcal{H}_{\mathcal{E}}}$ except for finitely many indices.

The operator algebra containing the observables of the total system is the von Neumann algebra

$$
\mathfrak{M}=\mathfrak{M}_{\mathcal{S}} \otimes \mathfrak{M}_{\mathcal{C}}
$$

\footnotetext{
${ }^{1}$ In other words, $\mathcal{H}_{\mathcal{C}}$ is obtained by taking the completion of the vector space of finite linear combinations of the form $\otimes_{m \geq 1} \phi_{m}$, where $\phi_{m} \in \mathcal{H}_{\mathcal{E}}, \phi_{m}=\psi_{\mathcal{E}}$ except for finitely many indices, in the norm induced by the inner product

$$
\left\langle\otimes_{m} \phi_{m}, \otimes_{m} \chi_{m}\right\rangle=\prod_{m}\left\langle\phi_{m}, \chi_{m}\right\rangle_{\mathcal{H}_{\mathcal{E}}} .
$$
}


which acts on the Hilbert space

$$
\mathcal{H}=\mathcal{H}_{\mathcal{S}} \otimes \mathcal{H}_{\mathcal{C}}
$$

The repeated interaction dynamics of observables in $\mathfrak{M}$ is characterized by an interaction time $0<\tau<\infty$ and a selfadjoint interaction operator

$$
V \in \mathfrak{M}_{\mathcal{S}} \otimes \mathfrak{M}_{\mathcal{E}}
$$

For times $t \in[\tau(m-1), \tau m)$, where $m \geq 1, \mathcal{S}$ interacts with the $m$-th element of the chain, while all other elements of the chain evolve freely (each one according to the dynamics $\alpha_{\mathcal{E}}^{t}$ ). The interaction of $\mathcal{S}$ with every element in the chain is the same (given by $V)$.

Let $L_{\mathcal{S}}$ and $L_{\mathcal{E}}$ be the standard Liouville operators ("positive temperature Hamiltonians", c.f. references of $[7,10])$, uniquely characterized by the following properties: $L_{\#}($ where $\#=\mathcal{S}, \mathcal{E})$ are selfadjoint operators on $\mathcal{H}_{\#}$ which implement the dynamics $\alpha_{\#}^{t}$,

$$
\alpha_{\#}^{t}(A)=\mathrm{e}^{\mathrm{i} t L_{\#}} A \mathrm{e}^{-\mathrm{i} t L_{\#}}, \quad \forall A \in \mathfrak{M}_{\#}, \forall t \in \mathbb{R}
$$

and satisfy

$$
L_{\#} \psi_{\#}=0 \text {. }
$$

We define the selfadjoint operator

$$
L=L_{\mathcal{S}}+L_{\mathcal{E}}+V,
$$

omitting trivial factors $\mathbb{1}_{\mathcal{S}}$ or $\mathbb{1}_{\mathcal{E}}$ (by $L_{\mathcal{S}}$ in $(2.9)$ we really mean $L_{\mathcal{S}} \otimes \mathbb{1}_{\mathcal{E}}$, etc). $L$ generates the group of $*$ automorphisms $\mathrm{e}^{\mathrm{i} t L} \cdot \mathrm{e}^{-\mathrm{i} t L}$ of $\mathfrak{M}_{\mathcal{S}} \otimes \mathfrak{M}_{\mathcal{E}}$, the interacting dynamics between $\mathcal{S}$ and an element $\mathcal{E}$ of the chain $\mathcal{C}$. The explicit form of the operator $V$ is dictated by the underlying physics, we give some examples in Section 4 .

For $m \geq 1$ let us denote by

$$
\widetilde{L}_{m}=L_{m}+\sum_{k \neq m} L_{\mathcal{E}, k}
$$

the generator of the total dynamics during the interval $[(m-1) \tau, m \tau)$. We have introduced $L_{m}$, the operator on $\mathcal{H}$ that acts trivially on all elements of the chain except for the $m$-th one. On the remaining part of the space (which is isomorphic to $\mathcal{H}_{\mathcal{S}} \otimes \mathcal{H}_{\mathcal{E}}$ ), $L_{m}$ acts as $L,(2.9)$. We have also set $L_{\mathcal{E}, k}$ to be the operator on $\mathcal{H}$ that acts nontrivially only on the $k$-th element of the chain, on which it equals $L_{\mathcal{E}}$. Of course, the infinite sum in (2.10) must be interpreted in the strong sense on $\mathcal{H}$.

Decompose $t \in \mathbb{R}_{+}$as

$$
t=m(t) \tau+s(t)
$$

where $m(t)$ is the integer measuring the number of complete interactions of duration $\tau$ the system $\mathcal{S}$ has undergone at time $t$, and where $0 \leq s(t)<\tau$. The repeated interaction dynamics of an observable $A \in \mathfrak{M}$ is defined by

$$
\alpha_{\mathrm{RI}}^{t}(A)=U_{\mathrm{RI}}(t)^{*} A U_{\mathrm{RI}}(t)
$$


where the unitary

$$
U_{\mathrm{RI}}(t)=\mathrm{e}^{-\mathrm{i} s(t) \widetilde{L}_{m(t)+1}} \mathrm{e}^{-\mathrm{i} \tau \widetilde{L}_{m(t)}} \cdots \mathrm{e}^{-\mathrm{i} \tau \widetilde{L}_{1}}
$$

defines the Schrödinger dynamics on $\mathcal{H}$. According to this dynamics, $\mathcal{S}$ interacts in succession, for a fixed duration $\tau$ and a fixed interaction $V$, with the first $m(t)$ elements of the chain, and for the remaining duration $s(t)$ with the $(m(t)+1)$-th element of the chain.

Our goal is to examine the large time behaviour of expectation values of certain observables in normal states $\varrho$ on $\mathfrak{M}$ (states given by a density matrix on $\mathcal{H}$ ). The system $\mathcal{S}$ feels an effective dynamics induced by the interaction with the chain $\mathcal{C}$. Under a suitable ergodicity assumption on this effective dynamics the small system is driven to an asymptotic state, as time increases. We will express the effective dynamics and the ergodic assumption using the modular data of the pair $\left(\mathfrak{M}_{\mathcal{S}} \otimes \mathfrak{M}_{\mathcal{C}}, \psi_{\mathcal{S}} \otimes \psi_{\mathcal{C}}\right)$.

Let $J$ and $\Delta$ denote the modular conjugation and the modular operator associated to $\left(\mathfrak{M}_{\mathcal{S}} \otimes \mathfrak{M}_{\mathcal{E}}, \psi_{\mathcal{S}} \otimes \psi_{\mathcal{E}}\right),[3]$. We assume that

(A) $\Delta^{1 / 2} V \Delta^{-1 / 2} \in \mathfrak{M}_{\mathcal{S}} \otimes \mathfrak{M}_{\mathcal{E}}$

and we introduce the operator

$$
K=L-J \Delta^{1 / 2} V \Delta^{-1 / 2} J,
$$

called the Liouville operator associated to $\psi_{\mathcal{S}} \otimes \psi_{\mathcal{E}}$, [7, 10]. It generates a strongly continuous group of bounded operators, denoted $\mathrm{e}^{\mathrm{i} t K}$, satisfying $\left\|\mathrm{e}^{\mathrm{i} t K}\right\| \leq \mathrm{e}^{|t|\left\|\Delta^{1 / 2} V \Delta^{-1 / 2}\right\|}$. The main feature of the operator $K$ is that $\mathrm{e}^{\mathrm{i} t K}$ implements the same dynamics as $\mathrm{e}^{\mathrm{i} t L}$ on $\mathfrak{M}_{\mathcal{S}} \otimes \mathfrak{M}_{\mathcal{E}}$ (since the difference $K-L$ belongs to the commutant $\mathfrak{M}_{\mathcal{S}}^{\prime} \otimes \mathfrak{M}_{\mathcal{E}}^{\prime}$ ), and that

$$
K \psi_{\mathcal{S}} \otimes \psi_{\mathcal{E}}=0 .
$$

Relation (2.15) follows from assumption (A), definition (2.14) and the properties

$$
\Delta^{-1 / 2} J=J \Delta^{1 / 2} \text { and } J \Delta^{1 / 2} A \psi_{\mathcal{S}} \otimes \psi_{\mathcal{E}}=A^{*} \psi_{\mathcal{S}} \otimes \psi_{\mathcal{E}},
$$

for any $A \in \mathfrak{M}_{\mathcal{S}} \otimes \mathfrak{M}_{\mathcal{E}} \cdot{ }^{2}$

Let

$$
P=\mathbb{1}_{\mathcal{H}_{\mathcal{S}}} \otimes\left|\psi_{\mathcal{C}}\right\rangle\left\langle\psi_{\mathcal{C}}\right|
$$

be the orthogonal projection onto $\mathcal{H}_{\mathcal{S}} \otimes \mathbb{C} \psi_{\mathcal{C}} \cong \mathcal{H}_{\mathcal{S}}$, where $\psi_{\mathcal{C}}$ is given in (2.2). Given an operator $B$ on $\mathcal{H}$, we identify $P B P$ with an operator acting on $\mathcal{H}_{\mathcal{S}}$. We have

Proposition 2.1 ([4]) There is a constant $C<\infty$ s.t. $\left\|\left(P \mathrm{e}^{\mathrm{i} t K} P\right)^{m}\right\|_{\mathcal{B}\left(\mathcal{H}_{\mathcal{S}}\right)} \leq C$, for all $t \in \mathbb{R}, m \geq 0$. In particular, $\operatorname{spec}\left(P \mathrm{e}^{\mathrm{i} t K} P\right) \subset\{z \in \mathbb{C}|| z \mid \leq 1\}$, and all eigenvalues lying on the unit circle are semisimple.

\footnotetext{
${ }^{2}$ The latter equation is the definition of $J$ and $\Delta$ : let $S$ be the the operator defined by $S A \psi_{\mathcal{S}} \otimes \psi_{\mathcal{C}}=$ $A^{*} \psi_{\mathcal{S}} \otimes \psi_{\mathcal{C}} . S$ is densely defined and closable. The polar decomposition of the closure of $S$ is $J \Delta^{1 / 2}$, it defines the anti-unitary involution $J$ and the positive operator $\Delta^{1 / 2}$.
} 
Relation (2.15) implies $P \mathrm{e}^{\mathrm{i} t K} P \psi_{\mathcal{S}}=\psi_{\mathcal{S}}$, for all $t \in \mathbb{R}$. Our assumption (E) on the effectiveness of the coupling is an ergodicity assumption on the discrete dynamics generated by

$$
M \equiv M(\tau)=P \mathrm{e}^{\mathrm{i} \tau K} P .
$$

(E) The spectrum of $M$ on the complex unit circle consists of the single eigenvalue $\{1\}$. This eigenvalue is simple (with corresponding eigenvector $\psi_{\mathcal{S}}$ ).

Assumption (E) guarantees that the adjoint operator $M^{*}$ has a unique invariant vector, called $\psi_{\mathcal{S}}^{*}\left(\right.$ normalized as $\left.\left\langle\psi_{\mathcal{S}}^{*}, \psi_{\mathcal{S}}\right\rangle=1\right)$, and that

$$
\lim _{m \rightarrow \infty} M^{m}=\pi:=\left|\psi_{\mathcal{S}}\right\rangle\left\langle\psi_{\mathcal{S}}^{*}\right|,
$$

in the operator sense, where $\pi$ is the rank one projection which projects onto $\mathbb{C} \psi_{\mathcal{S}}$ along $\left(\mathbb{C} \psi_{\mathcal{S}}^{*}\right)^{\perp}$. In fact, we have the following easy estimate (valid for any matrix $M$ with spectrum inside the unit disk and satisfying (E)).

Proposition $2.2([4])$ For any $\epsilon>0$ there exists a constant $C_{\epsilon}$ s.t. $\left\|M^{m}-\pi\right\| \leq$ $C_{\epsilon} \mathrm{e}^{-m(\gamma-\epsilon)}$, for all $m \geq 0$, where $\gamma:=\min _{z \in \operatorname{spec}(M) \backslash\{1\}}|\log | z||>0$.

The parameter $\gamma$ measures the speed of convergence. If all eigenvalues of $M$ are semisimple, then we have, in Proposition $2.2,\left\|M^{m}-\pi\right\| \leq C \mathrm{e}^{-m \gamma}$ for some constant $C$ and all $m \geq 0$.

As a last preparation towards an understanding of our results we discuss the kinds of observables we consider. One interesting class of observables is $\mathfrak{M}_{\mathcal{S}} \subset \mathfrak{M}$ which consists of observables of the system $\mathcal{S}$ only. There are other observables of interest. We may think of the system $\mathcal{S}$ as being fixed in space and of the chain as passing by $\mathcal{S}$ so that at the moment $t$, the $(m(t)+1)$-th element $\mathcal{E}$ is located near $\mathcal{S}$, c.f. $(2.11)$. A detector placed in the vicinity of $\mathcal{S}$ can measure at this moment in time observables of $\mathcal{S}$ and those of the $(m(t)+1)$-th element in the chain, i.e., an "instantaneous observable" of the form $A_{\mathcal{S}} \otimes \vartheta_{m(t)+1}\left(A_{\mathcal{E}}\right)$, where $A_{\mathcal{S}} \in \mathfrak{M}_{\mathcal{S}}, A_{\mathcal{E}} \in \mathfrak{M}_{\mathcal{E}}$, and $\vartheta_{m}: \mathfrak{M}_{\mathcal{E}} \rightarrow \mathfrak{M}_{\mathcal{C}}$ is defined by

$$
\vartheta_{m}\left(A_{\mathcal{E}}\right)=\mathbb{1}_{\mathcal{E}} \cdots \mathbb{1}_{\mathcal{E}} \otimes A_{\mathcal{E}} \otimes \mathbb{1}_{\mathcal{E}} \cdots
$$

the $A_{\mathcal{E}}$ on the right side of (2.19) acting on the $m$-th factor in the chain. An example of such an observable is the energy flux (variation) of the system $\mathcal{S}$. We call the operator $A_{\mathcal{S}} \otimes \vartheta_{m+1}\left(A_{\mathcal{E}}\right)$ an instantaneous observable determined by $A_{\mathcal{S}} \in \mathfrak{M}_{\mathcal{S}}$ and $A_{\mathcal{E}} \in \mathfrak{M}_{\mathcal{E}}$. One may consider more general observables, $[4,6]$.

\subsection{Results}

Throughout this section we assume that Conditions (A) and (E) of the previous section are satisfied. 


\subsection{Asymptotic state}

We consider the large time limit of expectations of instantaneous observables,

$$
E(t)=\varrho\left(\alpha_{\mathrm{RI}}^{t}\left(A_{\mathcal{S}} \otimes \vartheta_{m(t)+1}\left(A_{\mathcal{E}}\right)\right)\right)
$$

for normal initial states $\varrho$ on $\mathfrak{M}$. Define the state $\varrho_{+}$on $\mathfrak{M}_{\mathcal{S}}$ by

$$
\varrho_{+}\left(A_{\mathcal{S}}\right)=\left\langle\psi_{\mathcal{S}}^{*}, A_{\mathcal{S}} \psi_{\mathcal{S}}\right\rangle,
$$

where $\psi_{\mathcal{S}}^{*}$ is defined before $(2.18)$.

Theorem 2.3 ([4]) Suppose that conditions (A) and (E) are satisfied, and let $\varrho$ be a fixed normal state on $\mathfrak{M}$. For any $\epsilon>0$ there is a constant $C_{\epsilon}$ s.t. for all $t \geq 0$

$$
\left|E(t)-E_{+}(t)\right| \leq C_{\epsilon} \mathrm{e}^{-t(\gamma-\epsilon) / \tau},
$$

where $\gamma>0$ is given in Proposition 2.2, and where $E_{+}$is the $\tau$-periodic function

$$
E_{+}(t)=\varrho_{+}\left(P \alpha_{\mathrm{RI}}^{s(t)}\left(A_{\mathcal{S}} \otimes A_{\mathcal{E}}\right) P\right) .
$$

Remarks. 1) Using (2.22) and the uniqueness of the limit, one can see that the state $\varrho_{+}$does not depend on the choice of the reference state $\psi_{S}$.

2) $C_{\epsilon}$ in Theorem 2.3 is uniform in $\tau$ for $\tau>0$ varying in compact sets, and it is uniform in $\left\{A_{\mathcal{S}} \in \mathfrak{M}_{\mathcal{S}}, A_{\mathcal{E}} \subset \mathfrak{M}_{\mathcal{E}} \mid\left\|A_{\mathcal{S}}\right\|\left\|A_{\mathcal{E}}\right\| \leq\right.$ const. $\}$.

3) We refer to [4] for corresponding results for more general variables.

\subsection{Correlations \& reconstruction of initial state}

As Theorem 2.3 shows, the limit expectation values $E_{+}(t)$ are independent of the initial state (since the state $\varrho_{+}$is, c.f. (2.21)). However, limiting correlations are not, and their knowledge allows to reconstruct the initial state.

Fix a normal initial state $\varrho$ on $\mathfrak{M}$ and let $A \in \mathfrak{M}, A_{\mathcal{S}} \in \mathfrak{M}_{\mathcal{S}}, A_{\mathcal{E}} \in \mathfrak{M}_{\mathcal{E}}$. We define the correlation between $A$ and the instantaneous observable $A_{\mathcal{S}} \otimes \vartheta_{m(t)+1}\left(A_{\mathcal{E}}\right)$ by

$$
\mathcal{C}\left(t ; A, A_{\mathcal{S}}, A_{\mathcal{E}}\right)=\varrho\left(A \alpha_{\mathrm{RI}}^{t}\left(A_{\mathcal{S}} \otimes \vartheta_{m(t)+1}\left(A_{\mathcal{E}}\right)\right)\right) .
$$

Theorem 2.4 ([4]) For any $\epsilon>0$ there is a constant $C_{\epsilon}$ s.t. for all $t \geq 0$

$$
\left|\mathcal{C}\left(t ; A, A_{\mathcal{S}}, A_{\mathcal{E}}\right)-\mathcal{C}_{+}\left(t ; A, A_{\mathcal{S}}, A_{\mathcal{E}}\right)\right| \leq C_{\epsilon} \mathrm{e}^{-t(\gamma-\epsilon) / \tau},
$$

where $\gamma$ is given in Proposition 2.2, and where $\mathcal{C}_{+}$is the $\tau$-periodic limit correlation function

$$
\mathcal{C}_{+}\left(t ; A, A_{\mathcal{S}}, A_{\mathcal{E}}\right)=\varrho(A) \varrho_{+}\left(P \alpha_{\mathrm{RI}}^{s(t)}\left(A_{\mathcal{S}} \otimes A_{\mathcal{E}}\right) P\right),
$$

with $\varrho_{+}$defined in (2.21).

Relation (2.26) shows that the initial state $\varrho$ can be recovered from the knowledge of the asymptotic correlations $\mathcal{C}_{+}$and the asymptotic state $\varrho_{+}$. 


\section{Random systems}

\subsection{Dynamics and random matrix products}

We consider $\mathcal{S}$ to interact with a chain $\mathcal{C}=\mathcal{E}_{1}+\mathcal{E}_{2}+\cdots$, where the elements $\mathcal{E}_{k}$ may vary with $k$. Correspondingly, we have Hilbert spaces $\mathcal{H}_{\mathcal{S}}, \mathcal{H}_{\mathcal{E}_{k}}$, $\operatorname{dim} \mathcal{H}_{\mathcal{S}}<\infty, \operatorname{dim} \mathcal{H}_{\mathcal{E}_{k}} \leq \infty$ and von Neumann algebras of observables $\mathfrak{M}_{\mathcal{S}} \subset \mathcal{B}\left(\mathcal{H}_{\mathcal{E}}\right), \mathfrak{M}_{\mathcal{E}_{k}} \subset \mathcal{B}\left(\mathcal{H}_{\mathcal{E}_{k}}\right)$, describing $\mathcal{S}$ and $\mathcal{E}_{k}$, respectively. The uncoupled dynamics are given by groups of *automorphisms, $\alpha_{\mathcal{S}}^{t}, \alpha_{\mathcal{E}_{k}}^{t}$ of $\mathfrak{M}_{\mathcal{S}}, \mathfrak{M}_{\mathcal{E}_{k}}$. We introduce, as in the previous section, reference states $\psi_{\mathcal{S}} \in \mathcal{H}_{\mathcal{S}}$ and $\psi_{\mathcal{E}_{k}} \in \mathcal{H}_{\mathcal{E}_{k}}$, which are cyclic and separating vectors for the corresponding von Neumann algebras. Typical choices are KMS states with respect to the uncoupled dynamics, at given temperatures. The total Hilbert space is $\mathcal{H}=\mathcal{H}_{\mathcal{S}} \otimes \mathcal{H}_{\mathcal{C}}$, where $\mathcal{H}_{\mathcal{C}}=\otimes_{k \geq 1} \mathcal{H}_{\mathcal{E}_{k}}$, where the infinite tensor product is taken with respect to the vector $\psi_{0}=\psi_{\mathcal{S}} \otimes \psi_{\mathcal{C}}$, with $\psi_{\mathcal{C}}=\psi_{\mathcal{E}_{1}} \otimes \psi_{\mathcal{E}_{2}} \otimes \cdots$. The free dynamics is $\alpha_{\mathcal{S}}^{t} \otimes_{k \geq 1} \alpha_{\mathcal{E}_{k}}^{t}$, a group of *automorphisms on the von Neumann algebra $\mathfrak{M}=\mathfrak{M}_{\mathcal{S}} \otimes_{k \geq 1} \mathfrak{M}_{\mathcal{E}_{k}}$.

The interaction times are now given by $\tau_{1}, \tau_{2}, \ldots$, and the interaction operators by $V_{k} \in \mathfrak{M}_{\mathcal{S}} \otimes \mathfrak{M}_{\mathcal{E}_{k}}$. As in the previous section, there are self-adjoint Liouville operators $L_{\#}$, satisfying

$$
\mathrm{e}^{\mathrm{i} t L_{\#}} A_{\#} \mathrm{e}^{-\mathrm{i} t L_{\#}}=\alpha_{\#}^{t}\left(A_{\#}\right) \text {, and } L_{\#} \psi_{\#}=0,
$$

for all $t \in \mathbb{R}, A_{\#} \in \mathfrak{M}_{\#}$, where \# $=\mathcal{S}, \mathcal{E}_{k}$. We define the (discrete) repeated interaction Schrödinger dynamics of a state vector $\phi \in \mathcal{H}$, for $m \geq 0$, by

$$
U(m) \phi=\mathrm{e}^{-\mathrm{i} \widetilde{L}_{m}} \cdots \mathrm{e}^{-\mathrm{i} \widetilde{L}_{2}} \mathrm{e}^{-\mathrm{i} \widetilde{L}_{1}} \phi,
$$

where

$$
\widetilde{L}_{k}=\tau_{k} L_{k}+\tau_{k} \sum_{n \neq k} L_{\mathcal{E}_{n}}
$$

describes the dynamics during the time interval $\left[\tau_{1}+\cdots+\tau_{k-1}, \tau_{1}+\cdots+\tau_{k}\right)$, which corresponds to the time-step $k$ of the dynamical process. Here,

$$
L_{k}=L_{\mathcal{S}}+L_{\mathcal{E}_{k}}+V_{k}
$$

acts on $\mathcal{H}_{\mathcal{S}} \otimes \mathcal{H}_{\mathcal{E}_{k}}$. Of course, we understand that the operator $L_{\mathcal{E}_{n}}$ in (3.1) acts nontrivially only on the $n$-th factor of the Hilbert space $\mathcal{H}_{\mathcal{C}}$ of the chain.

Our goal is to understand the large-time asymptotics $(m \rightarrow \infty)$ of expectations

$$
\varrho\left(U(m)^{*} O U(m)\right)=\varrho\left(\alpha_{\mathrm{RI}}^{m}(O)\right),
$$

for normal states $\varrho$ and instantaneous observables $O$ (see before (2.19)). We denote the repeated interaction dynamics in this setting by

$$
\alpha_{\mathrm{RI}}^{m}(O)=U(m)^{*} O U(m) .
$$

Next, as in the previous section, we introduce new generators of the dynamics. Let $J_{m}$ and $\Delta_{m}$ denote the modular data of the pair $\left(\mathfrak{M}_{\mathcal{S}} \otimes \mathfrak{M}_{\mathcal{E}_{m}}, \psi_{\mathcal{S}} \otimes \psi_{\mathcal{E}_{m}}\right)$ (see e.g. [3]). In analogy with condition (A) (before (2.14)), we suppose that 
$\left(\mathbf{A}^{\prime}\right) \Delta_{m}^{1 / 2} V_{m} \Delta_{m}^{-1 / 2} \in \mathfrak{M}_{\mathcal{S}} \otimes \mathfrak{M}_{\mathcal{E}_{m}}, \quad \forall m \geq 1$

Let us define the Liouville operator $K_{m}$, compare with (2.14), by

$$
K_{m}=\tau_{m}\left[L_{\mathcal{S}}+L_{\mathcal{E}_{m}}+V_{m}-J_{m} \Delta_{m}^{1 / 2} V_{m} \Delta_{m}^{-1 / 2} J_{m}\right]
$$

Its main dynamical features,

$$
\begin{aligned}
\mathrm{e}^{\mathrm{i} \tau_{m} L_{m}} A \mathrm{e}^{-\mathrm{i} \tau_{m} L_{m}} & =\mathrm{e}^{\mathrm{i} K_{m}} A \mathrm{e}^{-\mathrm{i} K_{m}}, \quad \text { for } A \in \mathfrak{M}_{\mathcal{S}} \otimes \mathfrak{M}_{\mathcal{E}} \\
K_{m} \psi_{\mathcal{S}} \otimes \psi_{\mathcal{E}_{m}} & =0
\end{aligned}
$$

(see also (2.15)), are proven to hold by using standard relations of the modular data $\Delta_{m}, J_{m}$, see e.g. [4]. Note also the bound $\left\|\mathrm{e}^{ \pm i K_{m}}\right\| \leq \mathrm{e}^{\tau_{m} \| \Delta_{m}^{1 / 2} V_{m} \Delta_{m}^{-1 / 2}} \|$.

Denote by $P=\mathbb{1}_{\mathcal{S}} \otimes P_{\mathcal{C}}$ the projection onto the subspace $\mathcal{H}_{\mathcal{S}} \otimes \mathbb{C}\left[\psi_{\mathcal{E}_{1}} \otimes \psi_{\mathcal{E}_{2}} \otimes \cdots\right]$, and define, analogously to (2.17)

$$
M_{m}=P \mathrm{e}^{\mathrm{i} K_{m}} P
$$

which we identify with an operator on $\operatorname{Ran} P=\mathcal{H}_{\mathcal{S}}$. Suppose that we start initially in the state $\psi_{0}=\psi_{\mathcal{S}} \otimes \psi_{\mathcal{C}}$, where $\psi_{\mathcal{C}}=\psi_{\mathcal{E}_{1}} \otimes \psi_{\mathcal{E}_{2}} \otimes \cdots$. One shows that the expectation value of an observable $A \in \mathfrak{M}_{\mathcal{S}}$ at step $m$ is given by

$$
\left\langle\psi_{0}, \alpha_{\mathrm{RI}}^{m}(A) \psi_{0}\right\rangle=\left\langle\psi_{0}, M_{1} \cdots M_{m} A \psi_{0}\right\rangle,
$$

see the proof of Theorem 2.3 in Section 5 , and $[4,5]$. We thus see that in order to study the asymptotics of the dynamics, we must consider products $M_{1} \cdots M_{m}$. Two crucial properties of the operators $M_{k}$ are

1. $M_{1} \cdots M_{m}$ is bounded, uniformly in the number of factors $m$,

2. the $M_{k}$ have a common invariant vector, $M_{k} \psi_{\mathcal{S}}=\psi_{\mathcal{S}}$, for all $k$.

The former fact follows quite easily from the fact that the $M_{k}$ implement a dynamics which is norm-preserving, see e.g. $[4,5]$. The latter fact follows directly from the construction of the operators $M_{k}$. In the random setting, we shall consider the $M_{k}$ to be a family of independent, identically distributed random matrices, called $M(\omega)$.

\subsection{Results}

We introduce a general class of random matrices having the two properties mentioned above. Let $M(\omega)$ be a random matrix on $\mathbb{C}^{d}$, with probability space $(\Omega, \mathcal{F}, \mathrm{p})$. We say that $M(\omega)$ is a random reduced dynamics operator (RRDO) if

(1) There exists a norm $\|\cdot\| \|$ on $\mathbb{C}^{d}$ such that, for all $\omega, M(\omega)$ is a contraction on $\mathbb{C}^{d}$ endowed with the norm $\||\cdot|\|$.

(2) There is a vector $\psi_{\mathcal{S}}$, constant in $\omega$, such that $M(\omega) \psi_{\mathcal{S}}=\psi_{\mathcal{S}}$, for all $\omega$. 
Note that (1) is equivalent to the property 1 from in the previous section. We normalize $\psi_{\mathcal{S}}$ as $\left\|\psi_{\mathcal{S}}\right\|=1$, where $\|\cdot\|$ denotes the Euclidean norm. To an RRDO $M(\omega)$, we associate the (iid) random reduced dynamics process (RRDP)

$$
\Psi_{n}(\bar{\omega}):=M\left(\omega_{1}\right) \cdots M\left(\omega_{n}\right), \quad \bar{\omega} \in \Omega^{\mathbb{N}^{*}} .
$$

We will show that $\Psi_{n}$ has a decomposition into an exponentially decaying part and a fluctuating part. To identify these parts, we proceed as follows. It follows from (1) and (2) that the spectrum of an RRDO $M(\omega)$ must lie inside the closed complex unit disk, and that 1 is an eigenvalue (with eigenvector $\psi_{\mathcal{S}}$ ). Let $P_{1}(\omega)$ denote the spectral projection of $M(\omega)$ corresponding to the eigenvalue $1\left(\operatorname{dim} P_{1}(\omega) \geq 1\right)$, and let $P_{1}^{*}(\omega)$ be its adjoint operator. Define

$$
\psi(\omega):=P_{1}(\omega)^{*} \psi_{\mathcal{S}}
$$

and set

$$
P(\omega)=\left|\psi_{\mathcal{S}}\right\rangle\langle\psi(\omega)| .
$$

For $\psi, \phi \in \mathbb{C}^{d}$, we denote by $|\psi\rangle\langle\phi|$ the rank-one operator $|\psi\rangle\langle\phi| \chi=\langle\phi, \chi\rangle \psi$, and our convention is to take the inner products linear in the second factor. We put

$$
Q(\omega)=\mathbb{1}-P(\omega) .
$$

Note that the vector $\psi(\omega)$ is normalized as $\left\langle\psi_{\mathcal{S}}, \psi(\omega)\right\rangle=1$. We decompose $M(\omega)$ as

$$
M(\omega)=P(\omega)+Q(\omega) M(\omega) Q(\omega)=: P(\omega)+M_{Q}(\omega) .
$$

Taking into account this decomposition, one easily shows the following result.

Proposition 3.1 ([5]) We have

$$
\Psi_{n}(\bar{\omega}):=M\left(\omega_{1}\right) \cdots M\left(\omega_{n}\right)=\left|\psi_{\mathcal{S}}\right\rangle\left\langle\theta_{n}(\bar{\omega})\right|+M_{Q}\left(\omega_{1}\right) \cdots M_{Q}\left(\omega_{n}\right),
$$

where $\theta_{n}(\bar{\omega})$ is the Markov process

$$
\theta_{n}(\bar{\omega})=M^{*}\left(\omega_{n}\right) \cdots M^{*}\left(\omega_{2}\right) \psi\left(\omega_{1}\right),
$$

$M^{*}\left(\omega_{j}\right)$ being the adjoint operator of $M\left(\omega_{j}\right)$.

We analyze the two parts in the r.h.s. of (3.10) separately.

Definition Let $\mathcal{M}_{(E)}$ be the set of RRDOs $M$ whose spectrum on the complex unit circle consists only of a simple eigenvalue $\{1\}$.

On $\Omega^{\mathbb{N}^{*}}$ we define the probability measure $d \mathbb{P}$ in a standard fashion by

$$
\mathrm{d} \mathbb{P}=\Pi_{j \geq 1} \mathrm{dp}_{j}, \quad \text { where } \quad \mathrm{dp}_{j} \equiv \mathrm{dp}, \quad \forall j \in \mathbb{N}^{*} .
$$

Theorem 3.2 (Decaying process, [5]) Let $M(\omega)$ be a random reduced dynamics operator. Suppose that $\mathrm{p}\left(M(\omega) \in \mathcal{M}_{(E)}\right)>0$. Then there exist a set $\Omega_{1} \subset \Omega^{\mathbb{N}^{*}}$ and constants $C, \alpha>0$, s.t. $\mathbb{P}\left(\Omega_{1}\right)=1$ and s.t. for any $\bar{\omega} \in \Omega_{1}$ and any $n \geq 1$,

$$
\left\|M_{Q}\left(\omega_{1}\right) \cdots M_{Q}\left(\omega_{n}\right)\right\| \leq C \mathrm{e}^{-\alpha n}
$$


Remarks. 1. In the case where $M(\omega)=M$ is constant, and $M \in \mathcal{M}_{(E)}$, one readily shows that for any $\epsilon>0$ there is a $C_{\epsilon}$ such that $\left\|\left(M_{Q}\right)^{n}\right\| \leq C_{\epsilon} \mathrm{e}^{-n(\gamma-\epsilon)}$, for all $n \geq 0$, and where $\gamma=\min _{z \in \operatorname{spec}(M) \backslash\{1\}}|\log | z||$ (see e.g. also Proposition 2.2). It is remarkable that in the random case, the mere condition of $M$ having an arbitrarily small, nonvanishing probability to be in $\mathcal{M}_{(E)}$ suffices to guarantee the exponential decay of the product in (3.12).

2. Any stochastic matrix whose entries are all nonzero belongs to $\mathcal{M}_{(E)}$.

3. If $\{1\}$ is a simple eigenvalue of $M(\omega)$ then the decomposition (3.9) is just the spectral decomposition of the matrix $M(\omega)$.

4. The choice (3.8) ensures that $\psi(\omega)$ is an eigenvector of $M^{*}(\omega)$. Other choices of measurable $\psi(\omega)$ which are bounded in $\omega$ lead to different decompositions of $M(\omega)$, and can be useful as well. For instance, if $M(\omega)$ is a bistochastic matrix, then one can take for $\psi(\omega)$ an $M^{*}(\omega)$-invariant vector which is constant in $\omega$.

Our next result concerns the asymptotics of the Markov process (3.11). Set $\mathbb{E}[f]=$ $\int_{\Omega} f(\omega) \mathrm{dp}(\omega)$ for a random variable $f$, and denote by $P_{1, \mathbb{E}[M]}$ the spectral projection of $\mathbb{E}[M]$ onto the eigenvalue $\{1\}$.

Theorem 3.3 (Fluctuating process, [5]) Let $M(\omega)$ be a random reduced dynamics operator. Suppose that $\mathrm{p}\left(M(\omega) \in \mathcal{M}_{(E)}\right)>0$. Then we have $\mathbb{E}[M] \in \mathcal{M}_{(E)}$. Moreover, there exists a set $\Omega_{2} \subset \Omega^{\mathbb{N}^{*}}$ s.t. $\mathbb{P}\left(\Omega_{2}\right)=1$ and, for all $\bar{\omega} \in \Omega_{2}$,

$$
\lim _{N \rightarrow \infty} \frac{1}{N} \sum_{n=1}^{N} \theta_{n}(\bar{\omega})=\theta
$$

where

$$
\theta=\left(\mathbb{1}-\mathbb{E}\left[M_{Q}\right]^{*}\right)^{-1} \mathbb{E}[\psi]=P_{1, \mathbb{E}[M]}^{*} \mathbb{E}[\psi]=P_{1, \mathbb{E}[M]}^{*} \psi_{\mathcal{S}}
$$

Remarks. 5. In the case where $M$ is constant in $\omega$, we have $\mathbb{E}\left[M_{Q}\right]^{*}=\left(M_{Q}\right)^{*}, \mathbb{E}[\psi]=\psi$, and under the assumption of Theorem 3.3, that $M \in \mathcal{M}_{(E)}$. Therefore, $P_{1}=P=$ $\left|\psi_{\mathcal{S}}\right\rangle\langle\psi|$ and hence $Q^{*} \psi=0$, and $\left(M_{Q}\right)^{*} \psi=0$. Consequently, we have $\theta=\psi$. This coincides with the results of Theorem 2.3. However, the latter equality is not satisfied for general, $\omega$-dependent matrices $M$, as is shown in [5].

6 . The ergodic average limit of $\theta_{n}(\bar{\omega})$ does not depend on the particular choice of $\psi(\omega)$. This follows from the last equality in (3.14).

7. We show in [5] that for every fixed $\bar{\omega}, \theta_{n}(\bar{\omega})$ converges if and only if $\psi\left(\omega_{n}\right)$ converges, and that the limits coincide if they exist.

Combining Theorems 3.2 and 3.3 we obtain the following result.

Theorem 3.4 (Ergodic theorem for RRDP, [5]) Let $M(\omega)$ be a random reduced dynamics operator. Suppose $\mathrm{p}\left(M(\omega) \in \mathcal{M}_{(E)}\right)>0$. Then there exists a set $\Omega_{3} \subset \Omega^{\mathbb{N}^{*}}$ s.t. $\mathbb{P}\left(\Omega_{3}\right)=1$ and, for all $\bar{\omega} \in \Omega_{3}$,

$$
\lim _{N \rightarrow \infty} \frac{1}{N} \sum_{n=1}^{N} M\left(\omega_{1}\right) \cdots M\left(\omega_{n}\right)=\left|\psi_{\mathcal{S}}\right\rangle\langle\theta|=P_{1, \mathbb{E}[M]} .
$$


Remark. 8. If one can choose $\psi(\omega) \equiv \psi$ to be independent of $\omega$ (see also Remark 4 above), then one can show (see [5]) that $\theta_{n}(\bar{\omega})=\psi$, for all $n, \bar{\omega}$. It thus follows from (3.10)-(3.12) that $\lim _{n \rightarrow \infty} M\left(\omega_{1}\right) \cdots M\left(\omega_{n}\right)=\left|\psi_{\mathcal{S}}\right\rangle\langle\psi|$, a.s., exponentially fast.

\section{An example: spin systems}

We examine a model in which the small system as well as the elements of the chain are 2 -level systems. Consider the trace state on a two-level system, $\varrho_{\infty}(A)=\frac{1}{2} \operatorname{Tr}(A)$, where $A \in M_{2}(\mathbb{C})$ is a $2 \times 2$ matrix, and the trace is taken over $\mathbb{C}^{2}$. In order to represent $\varrho_{\infty}$ by a vector state, we must perform the Gelfand-Naimark-Segal construction. The representation Hilbert space is $\mathbb{C}^{2} \otimes \mathbb{C}^{2}$, and we have

$$
\varrho_{\infty}(A)=\left\langle\psi_{\infty},(A \otimes \mathbb{1}) \psi_{\infty}\right\rangle,
$$

where the inner product is that of $\mathbb{C}^{2} \otimes \mathbb{C}^{2}$ and where $\psi_{\infty}=\frac{1}{\sqrt{2}}\left[\varphi_{1} \otimes \varphi_{1}+\varphi_{2} \otimes \varphi_{2}\right]$, with

$$
\varphi_{1}=\left[\begin{array}{l}
1 \\
0
\end{array}\right], \text { and } \varphi_{2}=\left[\begin{array}{l}
0 \\
1
\end{array}\right] .
$$

This representation serves to represent the mixed state $\varrho_{\infty}$ by a vector state in an "enlarged" Hilbert space. The von Neumann algebra of observables for the small system and for the elements of the chain are

$$
\mathfrak{M}_{\mathcal{S}}=\mathfrak{M}_{\mathcal{E}}=M_{2}(\mathbb{C}) \otimes \mathbb{1}=\left\{A \otimes \mathbb{1} \mid A \in M_{2}(\mathbb{C})\right\},
$$

acting on the Hilbert space $\mathcal{H}_{\mathcal{S}}=\mathcal{H}_{\mathcal{E}}=\mathbb{C}^{2} \otimes \mathbb{C}^{2}$. Let $E_{\mathcal{S}}, E_{\mathcal{E}}>0$ be the "excited" energy levels of the small system and the elements of the chain, respectively. The dynamics are given by

$$
\alpha_{\mathcal{S}}^{t}(A \otimes \mathbb{1})=\mathrm{e}^{\mathrm{i} t h_{\mathcal{S}}} A \mathrm{e}^{-\mathrm{i} t h_{\mathcal{S}}} \otimes \mathbb{1}, \quad \text { and } \quad \alpha_{\mathcal{E}}^{t}(A \otimes \mathbb{1})=\mathrm{e}^{\mathrm{i} t h_{\mathcal{E}}} A \mathrm{e}^{-\mathrm{i} t h_{\mathcal{E}}} \otimes \mathbb{1},
$$

where

$$
h_{\mathcal{S}}=\left[\begin{array}{cc}
0 & 0 \\
0 & E_{\mathcal{S}}
\end{array}\right], \quad h_{\mathcal{E}}=\left[\begin{array}{cc}
0 & 0 \\
0 & E_{\mathcal{E}}
\end{array}\right]
$$

We choose the reference state $\psi_{\mathcal{S}}$ to be the tracial state $\psi_{\infty}$ defined above. The associated Liouville operator is $L_{\mathcal{S}}=h_{\mathcal{S}} \otimes \mathbb{1}-\mathbb{1} \otimes h_{\mathcal{S}}$, and the modular conjugation and modular operator associated to $\left(\mathfrak{M}_{\mathcal{S}}, \psi_{\mathcal{S}}\right)$ are

$$
J_{\mathcal{S}}(\phi \otimes \chi)=\chi \otimes \bar{\phi}, \quad \Delta_{\mathcal{S}}=\mathbb{1} \otimes \mathbb{1},
$$

where $\bar{\phi}$ denotes entrywise complex conjugation (in the canonical basis).

In order to avoid confusion between the small system and elements of the chain, we denote by $\phi_{i j}=\phi_{i} \otimes \phi_{j}$ the basis of $\mathcal{H}_{\mathcal{E}}$. We take the reference state of $\mathcal{E}$ to be the $\left(\alpha_{\mathcal{E}}^{t}, \beta\right)-\mathrm{KMS}$ state. Its representative vector is

$$
\psi_{\mathcal{E}}=\frac{1}{\sqrt{1+\mathrm{e}^{-\beta E_{\mathcal{E}}}}}\left(\phi_{11}+\mathrm{e}^{-\beta E_{\mathcal{E}} / 2} \phi_{22}\right)
$$


The standard Liouville operator is $L_{\mathcal{E}}=h_{\mathcal{E}} \otimes \mathbb{1}-\mathbb{1} \otimes h_{\mathcal{E}}$, and the modular conjugation and modular operator associated to $\left(\mathfrak{M}_{\mathcal{E}}, \psi_{\mathcal{E}}\right)$ are

$$
J_{\mathcal{E}}(\phi \otimes \chi)=\chi \otimes \bar{\phi}, \quad \Delta_{\mathcal{E}}=\mathrm{e}^{-\beta L_{\mathcal{E}}} .
$$

We now describe the interaction between $\mathcal{S}$ and $\mathcal{E}$. Let us denote by a and $\mathbf{a}^{*}$ the annihilation and creation operators,

$$
\mathbf{a}_{\#}=\left[\begin{array}{ll}
0 & 1 \\
0 & 0
\end{array}\right] \text { and } \mathbf{a}_{\#}^{*}=\left[\begin{array}{ll}
0 & 0 \\
1 & 0
\end{array}\right] \text {, }
$$

where $\#=\mathcal{S}, \mathcal{E}$. Let $a, b, c, d \in \mathbb{C}$ and set

$$
I=\left[\begin{array}{ll}
a & b \\
c & d
\end{array}\right]
$$

The interaction operator, acting on $\mathcal{H}_{\mathcal{S}} \otimes \mathcal{H}_{\mathcal{E}}=(\mathbb{C} \otimes \mathbb{C}) \otimes(\mathbb{C} \otimes \mathbb{C})$, is then defined as

$$
V=I \otimes \mathbb{1} \otimes \mathbf{a}^{*} \otimes \mathbb{1}+I^{*} \otimes \mathbb{1} \otimes \mathbf{a} \otimes \mathbb{1} .
$$

The standard Liouville operator, generating the interacting dynamics, is the self-adjoint operator

$$
L_{\lambda}:=L_{\mathcal{S}}+L_{\mathcal{E}}+\lambda V,
$$

where $\lambda$ is a real coupling constant. In this setting, we have $M_{\lambda=0}=P \mathrm{e}^{\mathrm{i} \tau L_{\mathcal{S}}} P$. Note that the spectrum of $M_{\lambda=0}$ is $\left\{\mathrm{e}^{-\mathrm{i} \tau E_{\mathcal{S}}}, 1,1, \mathrm{e}^{\mathrm{i} \tau E_{\mathcal{S}}}\right\}$.

Deterministic spin model. We assume that

(S0) $\tau E_{\mathcal{S}} \notin \pi \mathbb{Z}$,

in order to ensure that the spectrum of $M_{\lambda=0}$ does not collapse, i.e., that $\mathrm{e}^{-\mathrm{i} \tau E_{\mathcal{S}}} \neq \mathrm{e}^{\mathrm{i} \tau E_{\mathcal{S}}}$ and $\mathrm{e}^{ \pm \mathrm{i} \tau E_{\mathcal{S}}} \neq 1$. This assumption is made for convenience, and can most probably be eliminated.

The operator $K_{\lambda}$ associated to $L_{\lambda}$ and to the reference state $\psi_{\mathcal{S}} \otimes \psi_{\mathcal{E}} \otimes \psi_{\mathcal{E}} \cdots$ (see $(2.14))$ is

$$
K_{\lambda}:=L_{\mathcal{S}}+L_{\mathcal{E}}+\lambda\left(V-J \Delta^{1 / 2} V \Delta^{-1 / 2} J\right)=K_{0}+\lambda W .
$$

We consider the following assumptions on the effectiveness of the coupling operator $I$, (4.6), and where $\tau$ denotes the interaction time.

(S1) $b \neq 0$ and $\tau\left(E_{\mathcal{E}}-E_{\mathcal{S}}\right) \notin 2 \pi \mathbb{Z} \backslash\{0\}$.

(S2) $c \neq 0$ and $\tau\left(E_{\mathcal{E}}+E_{\mathcal{S}}\right) \notin 2 \pi \mathbb{Z} \backslash\{0\}$.

(S3) $a \neq d$ and $\tau E_{\mathcal{E}} \notin 2 \pi \mathbb{Z} \backslash\{0\}$.

The proof of the following result is based on peturbation theory in $\lambda$. We outline it in Section 5. Set $\operatorname{sinc}(x)=\sin (x) / x$. 
Theorem 4.1 (Deterministic spin model [4]) Suppose that either Assumption (S1), (S2) or (S3) is satisfied, and that (SO) holds. Then there exists $\lambda_{0}>0$ such that for all $0<|\lambda|<\lambda_{0}$, the operator $M_{\lambda}:=P \mathrm{e}^{\mathrm{i} \tau K_{\lambda}} P$ satisfies the ergodic assumption (E). In particular, Theorem 2.3 holds for this system, with $\gamma \geq \gamma_{0} \lambda^{2}+O\left(\lambda^{4}\right)$. Moreover, the asymptotic state $\varrho_{+, \lambda}$ is given by

$$
\varrho_{+, \lambda}\left(A_{\mathcal{S}}\right)=\frac{\alpha_{1}}{\alpha_{1}+\alpha_{2}}\left\langle\varphi_{1}, A_{\mathcal{S}} \varphi_{1}\right\rangle+\frac{\alpha_{2}}{\alpha_{1}+\alpha_{2}}\left\langle\varphi_{2}, A_{\mathcal{S}} \varphi_{2}\right\rangle+O\left(\lambda^{2}\right),
$$

where

$$
\begin{aligned}
& \alpha_{1}:=|b|^{2} \operatorname{sinc}^{2}\left[\frac{\tau\left(E_{\mathcal{E}}-E_{\mathcal{S}}\right)}{2}\right]+\mathrm{e}^{-\beta E_{\mathcal{E}}}|c|^{2} \operatorname{sinc}^{2}\left[\frac{\tau\left(E_{\mathcal{E}}+E_{\mathcal{S}}\right)}{2}\right], \\
& \alpha_{2}:=\mathrm{e}^{-\beta E_{\mathcal{E}}}|b|^{2} \operatorname{sinc}^{2}\left[\frac{\tau\left(E_{\mathcal{E}}-E_{\mathcal{S}}\right)}{2}\right]+|c|^{2} \operatorname{sinc}^{2}\left[\frac{\tau\left(E_{\mathcal{E}}+E_{\mathcal{S}}\right)}{2}\right], \\
& \gamma_{0}:=\tau^{2} \min \left\{\frac{\alpha_{1}+\alpha_{2}}{1+\mathrm{e}^{-\beta E_{\mathcal{E}}}}, \frac{1}{2} \frac{\alpha_{1}+\alpha_{2}}{1+\mathrm{e}^{-\beta E_{\mathcal{E}}}}+\frac{|a-d|^{2}}{2} \operatorname{sinc}^{2}\left(\frac{\tau E_{\mathcal{E}}}{2}\right)\right\} .
\end{aligned}
$$

Assumptions (S1), (S2), (S3) serve to ensure that $\gamma_{0}>0$. Indeed, if (S1) is satisfied, then $\alpha_{1}>0$, if (S2) is satisfied, then $\alpha_{2}>0$, and if (S3) holds, then $\frac{|a-d|^{2}}{2} \operatorname{sinc}^{2}\left(\frac{\tau E_{\mathcal{E}}}{2}\right)>$ 0 .

Random spin model. We take the same system with a random interaction time $\tau=\tau(\omega)$ and random temperature of the chain $\beta=\beta(\omega)$. We suppose that $\tau(\omega) \in\left[\tau_{\min }, \tau_{\max }\right]$ for some $0<\tau_{\min }<\tau_{\max }<\infty$, and that $\beta(\omega) \in\left(0, \beta_{\max }\right]$ for some $0<\beta_{\max }<\infty$. (The following analysis extends to more general settings, where e.g. also the energies $E_{\mathcal{E}}, E_{\mathcal{S}}$, or the coefficients $a, b, c, d$, of the interaction matrix are random). The above conditions (S0)-(S4) are replaced by

(R0) There is a $\delta_{\mathcal{S}}>0$ s.t. $\mathrm{p}\left(\operatorname{dist}\left(\tau E_{\mathcal{S}}, \pi \mathbb{Z}\right)>\delta_{\mathcal{S}}\right) \neq 0$.

(R1) $b \neq 0$, and there is a $\delta_{-}>0$ s.t. $\mathrm{p}\left(\operatorname{dist}\left(\frac{\tau}{2}\left(E_{\mathcal{E}}-E_{\mathcal{S}}\right), \pi \mathbb{Z} \backslash\{0\}\right)>\delta_{-}\right) \neq 0$.

(R2) $c \neq 0$, and there is a $\delta_{+}>0$ s.t. $\mathrm{p}\left(\operatorname{dist}\left(\frac{\tau}{2}\left(E_{\mathcal{E}}+E_{\mathcal{S}}\right), \pi \mathbb{Z} \backslash\{0\}\right)>\delta_{+}\right) \neq 0$.

(R3) $a \neq d$, and there is a $\delta_{\mathcal{E}}>0$ s.t. $\mathrm{p}\left(\operatorname{dist}\left(\frac{\tau}{2} E_{\mathcal{E}}, \pi \mathbb{Z} \backslash\{0\}\right)>\delta_{\mathcal{E}}\right) \neq 0$.

Remark. These conditions can be rephrased; for instance, (R0) is equivalent to: $\mathrm{p}\left(\tau E_{\mathcal{E}} \in \pi \mathbb{Z}\right) \neq 1$ (and similarly for (R1)-(R3)).

We introduce the following parameters:

$$
\begin{aligned}
C & =2\|I\|\left(1+\mathrm{e}^{\beta_{\max } E_{\mathcal{E}} / 2}\right) \\
D & =100 \tau_{\max }^{2} C^{4} \cosh \left(\tau_{\max } C\right)\left[1+\frac{1+\tau_{\max }^{2} C^{2} \cosh \left(\tau_{\max } C\right)}{\sqrt{1-\cos \delta_{\mathcal{S}}}}\right] \\
\lambda_{1} & =\frac{|b|}{\sqrt{D}} \min \left\{1, \frac{2\left|\sin \left(\delta_{-}\right)\right|}{\left|E_{\mathcal{E}}-E_{\mathcal{S}}\right| \tau_{\min }}\right\} \\
\lambda_{2} & =\frac{|c|}{\sqrt{D}} \min \left\{1, \frac{2\left|\sin \left(\delta_{+}\right)\right|}{\left|E_{\mathcal{E}}-E_{\mathcal{S}}\right| \tau_{\min }}\right\} \\
\lambda_{3} & =\frac{|a-d|}{\sqrt{D}} \min \left\{1, \frac{2\left|\sin \left(\delta_{\mathcal{E}}\right)\right|}{\left|E_{\mathcal{E}}\right| \tau_{\min }}\right\}
\end{aligned}
$$


Theorem 4.2 (Random spin model) Suppose that (RO) holds, and that for one $j=$ $1, j=2$ or $j=3$, the following holds: (Rj) is satisfied and $0<|\lambda|<\lambda_{j}$. Then the results of Theorems 3.2-3.4 hold.

We prove this result in Section 5 .

\section{Some proofs}

Outline of the proof of Theorem 2.3. We outline the proof for the special case where $\varrho_{+}(\cdot)=\left\langle\psi_{0}, \cdot \psi_{0}\right\rangle$, where $\psi_{0}=\psi_{\mathcal{S}} \otimes_{k \geq 1} \psi_{\mathcal{E}}$, and for observables $A_{\mathcal{S}} \in \mathfrak{M}_{\mathcal{S}}$. Recall that

$$
\alpha_{\mathrm{RI}}^{t}\left(A_{\mathcal{S}}\right)=\mathrm{e}^{\mathrm{i} \tau \widetilde{L}_{1}} \cdots \mathrm{e}^{\mathrm{i} \tau \widetilde{L}_{m}} \mathrm{e}^{\mathrm{i} s \widetilde{L}_{m+1}} A_{\mathcal{S}} \mathrm{e}^{-\mathrm{i} s \widetilde{L}_{m+1}} \mathrm{e}^{-\mathrm{i} \tau \widetilde{L}_{m}} \cdots \mathrm{e}^{-\mathrm{i} \tau \widetilde{L}_{1}}
$$

The proof of Theorem 2.3 has four main ingredients.

1. Factorization of the free dynamics. Taking into account the decomposition (2.10) of $\widetilde{L}_{k}$, we see that

$$
\mathrm{e}^{-\mathrm{i} s \widetilde{L}_{m+1}} \mathrm{e}^{-\mathrm{i} \tau \widetilde{L}_{m}} \cdots \mathrm{e}^{-\mathrm{i} \tau \widetilde{L}_{1}}=U_{m}^{-} \mathrm{e}^{-\mathrm{i} s L_{m+1}} \mathrm{e}^{-\mathrm{i} \tau L_{m}} \cdots \mathrm{e}^{-\mathrm{i} \tau L_{1}} U_{m}^{+},
$$

where $U_{m}^{ \pm}$are the unitaries

$$
\begin{aligned}
& U_{m}^{-}=\exp \left[-\mathrm{i} \sum_{j=1}^{m}[(m-j) \tau+s] L_{\mathcal{E}, j}\right], \\
& U_{m}^{+}=\exp \left[-\mathrm{i} \sum_{j=2}^{m+1}(j-1) \tau L_{\mathcal{E}, j}-\mathrm{i}(m \tau+s) \sum_{j \geq m+1} L_{\mathcal{E}, j}\right] .
\end{aligned}
$$

Clearly, $U_{m}^{ \pm} \psi_{0}=\psi_{0}$, for all $m$, so (5.1) gives

$$
\left\langle\psi_{0}, \alpha_{\mathrm{RI}}^{t}\left(A_{\mathcal{S}}\right) \psi_{0}\right\rangle=\left\langle\psi_{0}, \mathrm{e}^{\mathrm{i} \tau L_{1}} \cdots \mathrm{e}^{\mathrm{i} s L_{m+1}} A_{\mathcal{S}} \mathrm{e}^{-\mathrm{i} s L_{m+1}} \cdots \mathrm{e}^{-\mathrm{i} \tau L_{1}} \psi_{0}\right\rangle .
$$

2. Passage to non-self-adjoint generator of dynamics. We now employ a trick that has been recently invented to analyze the asymptotics of open quantum systems far from equilibrium [7,10]. We replace the operators $L_{m}$ by operators $K_{m}$, having the property that $K_{m}$ implements the same dynamics as $L_{m}$, but satisfies in addition the property $K_{m} \psi_{0}=0$. The existence of such operators is linked to the deep Tomita-Takesaki theory of von Neumann algebras, and in fact, $K_{m}$ is expressed in terms of the modular data $(J, \Delta)$ associated to the pair $\left(\mathfrak{M}, \psi_{0}\right)$, [3]. It is given explicitly by (3.9). We thus obtain from (5.2)

$$
\left\langle\psi_{0}, \alpha_{\mathrm{RI}}^{t}\left(A_{\mathcal{S}}\right) \psi_{0}\right\rangle=\left\langle\psi_{0}, \mathrm{e}^{\mathrm{i} \tau K_{1}} \cdots \mathrm{e}^{\mathrm{i} \tau K_{m}} \mathrm{e}^{\mathrm{i} s K_{m+1}} A_{\mathcal{S}} \psi_{0}\right\rangle .
$$

3. Reduction of the dynamics. In this step we take advantage of the fact that the elements $\mathcal{E}$ in the chain $\mathcal{C}$ are independently prepared (not entangled) and dynamically not directly coupled. Let $P$ be the orthogonal projection onto $\mathcal{H}_{\mathcal{S}} \otimes$ 
$\mathbb{C} \psi_{\mathcal{C}}$, where $\psi_{\mathcal{C}}=\psi_{\mathcal{E}} \otimes \psi_{\mathcal{E}} \otimes \cdots$. Then $A_{\mathcal{S}} \psi_{0}=A_{\mathcal{S}} P \psi_{0}=P A_{\mathcal{S}} \psi_{0}$ (since $\psi_{0}=P \psi_{0}$, and $\left.A_{\mathcal{S}} \in \mathfrak{M}_{\mathcal{S}}\right)$, so we are led to consider $P \mathrm{e}^{\mathrm{i} \tau K_{1}} \cdots \mathrm{e}^{\mathrm{i} \tau K_{m}} \mathrm{e}^{\mathrm{i} s K_{m+1}} P$ in (5.3). Writing $P=P_{\psi_{\mathcal{E}}} \otimes P_{\psi_{\mathcal{E}}} \otimes \cdots$, where $\psi_{\mathcal{E}}=\left|\psi_{\mathcal{E}}\right\rangle\left\langle\psi_{\mathcal{E}}\right|$, we note that

$$
\mathrm{e}^{\mathrm{i} s K_{m+1}} P=\left(P_{\psi_{\mathcal{E}}} \otimes \cdots P_{\psi_{\mathcal{E}}} \otimes P_{\psi_{\mathcal{E}}} \otimes \mathbb{1} \otimes P_{\psi_{\mathcal{E}}} \otimes \cdots\right) \mathrm{e}^{\mathrm{i} s K_{m+1}} P
$$

where the identity operator stands at the spot $(m+1)$. This is true simply because $K_{m+1}$ acts non-trivially only on the factor $(m+1)$ on the chain. On the other hand, for the same reason, we have

$$
P \mathrm{e}^{\mathrm{i} \tau K_{1}} \cdots \mathrm{e}^{\mathrm{i} \tau K_{m}}=P \mathrm{e}^{\mathrm{i} \tau K_{1}} \cdots \mathrm{e}^{\mathrm{i} \tau K_{m}}\left(\mathbb{1} \otimes \mathbb{1} \cdots \otimes \mathbb{1} \otimes P_{\psi_{\mathcal{E}}} \otimes P_{\psi_{\mathcal{E}}} \otimes \cdots\right),
$$

where the first nontrivial projection on the right is on factor $m+1$. The combination of (5.4) and (5.5) gives

$$
P \mathrm{e}^{\mathrm{i} \tau K_{1}} \cdots \mathrm{e}^{\mathrm{i} \tau K_{m}} \mathrm{e}^{\mathrm{i} s K_{m+1}} P=P \mathrm{e}^{\mathrm{i} \tau K_{1}} P \cdots P \mathrm{e}^{\mathrm{i} \tau K_{m}} P \mathrm{e}^{\mathrm{i} s K_{m+1}} P
$$

Since the interaction is the same at each step in the repeated interaction process, we can identify the operator

$$
P \mathrm{e}^{\mathrm{i} \tau K_{k}} P \equiv M
$$

as an operator on $\mathcal{H}_{\mathcal{S}}$, independent of $k$. Thus, with (5.3) and (5.6), we obtain

$$
\left\langle\psi_{0}, \alpha_{\mathrm{RI}}^{t}\left(A_{\mathcal{S}}\right) \psi_{0}\right\rangle=\left\langle\psi_{0}, M^{m(t)} P \mathrm{e}^{\mathrm{i} s(t) K} P A_{\mathcal{S}} \psi_{0}\right\rangle,
$$

where we also set $P \mathrm{e}^{\mathrm{i} s(t) K_{k}} P=P \mathrm{e}^{\mathrm{i} s(t) K} P$, for any $k$. The dynamical process is now clear: the term $M^{m(t)}$ will have a limit as $t \rightarrow \infty$ (under suitable conditions), while $P \mathrm{e}^{\mathrm{i} s(t) K} P$ is oscillating in $t$ (with period $\tau$ ).

4. Spectral analysis of $M$ and decay of $M^{m(t)}$. Proposition 2.2 and equation (5.7) show that

$$
\left|\left\langle\psi_{0}, \alpha_{\mathrm{RI}}^{t}\left(A_{\mathcal{S}}\right) \psi_{0}\right\rangle-\left\langle\psi_{\mathcal{S}}^{*}, \alpha_{\mathrm{RI}}^{s(t)}\left(A_{\mathcal{S}}\right) \psi_{\mathcal{S}}\right\rangle\right| \leq C_{\epsilon} \mathrm{e}^{-t(\gamma-\epsilon) / \tau}
$$

where we have taken into account that

$$
\left\langle\psi_{0}, P \mathrm{e}^{\mathrm{i} s(t) K} P A_{\mathcal{S}} \psi_{0}\right\rangle=\left\langle\psi_{0}, P \mathrm{e}^{\mathrm{i} s(t) L} A_{\mathcal{S}} \mathrm{e}^{-\mathrm{i} s(t) L} \psi_{0}\right\rangle=\left\langle\psi_{0}, \alpha_{\mathrm{RI}}^{s(t)}\left(A_{\mathcal{S}}\right) \psi_{0}\right\rangle .
$$

This concludes the proof of Theorem 3.2 in the special setting.

Outline of the proof of Theorem 4.1. A Dyson series expansion gives

$$
\begin{aligned}
\mathrm{e}^{\mathrm{i} \tau K_{\lambda}}= & \mathrm{e}^{\mathrm{i} \tau K_{0}}+\mathrm{i} \lambda \int_{0}^{\tau} \mathrm{d} t \mathrm{e}^{\mathrm{i}(\tau-t) K_{0}} W \mathrm{e}^{\mathrm{i} t K_{0}} \\
& \quad-\lambda^{2} \int_{0}^{\tau} \int_{0}^{t} \mathrm{e}^{\mathrm{i}(\tau-t) K_{0}} W \mathrm{e}^{\mathrm{i}(t-s) K_{0}} W \mathrm{e}^{\mathrm{i} s K_{0}} \mathrm{~d} s \mathrm{~d} t+R(\tau, \lambda),
\end{aligned}
$$


where

$$
\begin{aligned}
& R(\tau, \lambda) \\
& =\sum_{n \geq 2}(-\lambda)^{2 n} \int_{0}^{\tau} \mathrm{d} t_{1} \cdots \int_{0}^{t_{2 n-1}} \mathrm{~d} t_{2 n} \mathrm{e}^{\mathrm{i} t_{1} K_{0}} W \mathrm{e}^{-\mathrm{i} t_{1} K_{0}} \cdots \mathrm{e}^{\mathrm{i} t_{2 n} K_{0}} W \mathrm{e}^{-\mathrm{i} t_{2 n} K_{0}} .
\end{aligned}
$$

After a somewhat lengthy but straightforward computation, one obtains a perturbative expression for the operator $M(\lambda)=P \mathrm{e}^{\mathrm{i} \tau K_{\lambda}} P$, and the follwing expansions for the three eigenvalues $e_{0}(\lambda), e_{ \pm}(\lambda)$ of $M(\lambda)$, other than the eigenvalue 1, see [4], Section 4.9:

$$
\begin{aligned}
e_{0}(\lambda)= & 1-\frac{\lambda^{2} \tau^{2}}{1+\mathrm{e}^{-\beta E_{\mathcal{E}}}}\left(\alpha_{1}+\alpha_{2}\right)+O\left(\lambda^{4}\right) \\
e_{-}(\lambda)=\frac{\lambda^{2} \tau^{2}}{e_{+}(\lambda)} & \left(\alpha_{1}+\alpha_{2}+\left(1+\mathrm{e}^{-\beta E_{\mathcal{E}}}\right)|a-d|^{2} \operatorname{sinc}^{2}\left(\frac{\tau E_{\mathcal{E}}}{2}\right)\right) \\
e_{+}(\lambda)=\mathrm{e}^{\mathrm{i} \tau E_{\mathcal{S}}}\left[1-\frac{\lambda^{2} \tau^{2}}{2\left(1+\mathrm{e}^{-\beta E_{\mathcal{E}}}\right)}\right. & \left(\left(1-\mathrm{e}^{-\beta E_{\mathcal{E}}}\right)\left(|a|^{2}-|d|^{2}\right) \frac{1-\operatorname{sinc}\left(\tau E_{\mathcal{E}}\right)}{\tau E_{\mathcal{E}}}\right. \\
+\mathrm{i} \frac{\mathrm{e}^{-\beta E_{\mathcal{E}}}}{1+\operatorname{sinc}^{2}\left(\frac{\tau E_{\mathcal{E}}}{2}\right)} & \left.+\left(1-\mathrm{e}^{-\beta E_{\mathcal{E}}}\right) \operatorname{Im}(\bar{a} d) \operatorname{sinc}^{-\beta E_{\mathcal{E}}}\right)|b|^{2} \frac{\left.1-\operatorname{sinc}\left(E_{\mathcal{E}}-E_{\mathcal{S}}\right)\right)}{\tau\left(E_{\mathcal{E}}-E_{\mathcal{S}}\right)} \\
& \left.\left.-\left(1+\mathrm{e}^{-\beta E_{\mathcal{E}}}\right)|c|^{2} \frac{1-\operatorname{sinc}\left(\tau\left(E_{\mathcal{E}}+E_{\mathcal{S}}\right)\right)}{\tau\left(E_{\mathcal{E}}+E_{\mathcal{S}}\right)}\right)\right]+O\left(\lambda^{4}\right) .
\end{aligned}
$$

These expressions show that we have

$$
\begin{aligned}
&|\log | e_{0}(\lambda)|| \geq \frac{\lambda^{2} \tau^{2}}{1+\mathrm{e}^{-\beta E_{\mathcal{E}}}}\left(\alpha_{1}+\alpha_{2}\right)+O\left(\lambda^{4}\right), \\
&|\log | e_{ \pm}(\lambda)|| \geq \frac{\lambda^{2} \tau^{2}}{2\left(1+\mathrm{e}^{\left.-\beta E_{\mathcal{E}}\right)}\right.}\left[\alpha_{1}+\alpha_{2}+\left(1+\mathrm{e}^{-\beta E_{\mathcal{E}}}\right)|a-d|^{2} \operatorname{sinc}^{2}\left(\frac{\tau E_{\mathcal{E}}}{2}\right)\right]+O\left(\lambda^{4}\right) .
\end{aligned}
$$

Therefore, $\min _{z \in \operatorname{spec}(M) \backslash\{1\}}|\log | z|| \geq \lambda^{2} \gamma_{0}+O\left(\lambda^{4}\right)$, where $\gamma_{0}$ is as in Theorem 4.1.

In order to calculate the asymptotic state $\varrho_{+, \lambda},(4.10)$, one performs perturbation theory in the formula (2.21). We do not present the calculations here, see [4].

Outline of proof of Theorem 4.2. We first get the following deterministic result.

Lemma 5.1 Define

$$
\left(\lambda_{0}\right)^{2}=\frac{|b|^{2} \operatorname{sinc}^{2}\left[\frac{\tau\left(E_{\mathcal{E}}-E_{\mathcal{S}}\right)}{2}\right]+|c|^{2} \operatorname{sinc}^{2}\left[\frac{\tau\left(E_{\mathcal{E}}+E_{\mathcal{S}}\right)}{2}\right]+|a-d|^{2} \operatorname{sinc}^{2}\left[\frac{\tau E_{\mathcal{E}}}{2}\right]}{100 \tau^{2}\|W\|^{4} \cosh ^{2}(\tau\|W\|)\left\{1+r_{0}^{-1}(\tau)\left[1+\tau^{2}\|W\|^{2} \cosh (\tau\|W\|)\right]\right\}},
$$

where $r_{0}^{2}(\tau)=2 \min \left\{1-\cos \left(\tau E_{\mathcal{S}}\right), 1-\cos \left(2 \tau E_{\mathcal{S}}\right)\right\}$. If $0<|\lambda|<\min \left\{1, \lambda_{0}\right\}$, then $M_{\lambda} \in \mathcal{M}_{(E)}$. Moreover, we have in this case

$$
\begin{aligned}
& \left|e_{\#}(\lambda)\right|< \\
& \quad 1-\frac{\lambda^{2} \tau^{2}}{8}\left\{|b|^{2} \operatorname{sinc}^{2}\left[\frac{\tau\left(E_{\mathcal{E}}-E_{\mathcal{S}}\right)}{2}\right]+|c|^{2} \operatorname{sinc}^{2}\left[\frac{\tau\left(E_{\mathcal{E}}+E_{\mathcal{S}}\right)}{2}\right]+|a-d|^{2} \operatorname{sinc}^{2}\left[\frac{\tau E_{\mathcal{E}}}{2}\right]\right\},
\end{aligned}
$$


where $\#= \pm$, and

$$
\left|e_{0}(\lambda)\right|<1-\frac{\lambda^{2} \tau^{2}}{2}\left\{|b|^{2} \operatorname{sinc}^{2}\left[\frac{\tau\left(E_{\mathcal{E}}-E_{\mathcal{S}}\right)}{2}\right]+|c|^{2} \operatorname{sinc}^{2}\left[\frac{\tau\left(E_{\mathcal{E}}+E_{\mathcal{S}}\right)}{2}\right]\right\} .
$$

The proof of this lemma is a straightforward perturbation theory argument, in which remainders are carefully estimated, see e.g. [8], Chapter II. To prove Theorem 4.2, we need to show that

$$
\mathrm{p}\left(M(\omega) \in \mathcal{M}_{(E)}\right)>0 .
$$

Let us consider the case where (R0) and (R1) are satisfied, and $0<|\lambda|<\lambda_{1}$. The other cases are dealt with in the same manner. We see from the definition of $\lambda_{0}$ given in Lemma 5.1 that

$$
\left(\lambda_{0}\right)^{2} \geq \frac{|b|^{2} \operatorname{sinc}^{2}\left[\frac{\tau\left(E_{\mathcal{E}}-E_{\mathcal{S}}\right)}{2}\right]}{100 \tau^{2}\|W\|^{4} \cosh ^{2}(\tau\|W\|)\left\{1+r_{0}^{-1}(\tau)\left[1+\tau^{2}\|W\|^{2} \cosh (\tau\|W\|)\right]\right\}} .
$$

Recall the definition of $\lambda_{1}$ given before Theorem 4.2. Let $\Omega_{1}$ denote the set of $\omega$ s.t. $\operatorname{dist}\left(\frac{\tau}{2}\left(E_{\mathcal{E}}-E_{\mathcal{S}}\right), \pi \mathbb{Z} \backslash\{0\}\right)>\delta_{-}$and s.t. $\operatorname{dist}\left(\tau E_{\mathcal{S}}, \pi \mathbb{Z}\right)>\delta_{\mathcal{S}} . \Omega_{1}$ has measure one, and for each $\omega \in \Omega_{1}$ the r.h.s. of (5.12) is bounded from below by $\lambda_{1}^{2}$. This is readily seen by using that $\tau(\omega) \in\left[\tau_{\min }, \tau_{\max }\right], \beta(\omega) \in\left(0, \beta_{\max }\right]$, and that $\|W\| \leq 2\|I\|\left(1+\mathrm{e}^{\beta_{\max } E_{\mathcal{E}} / 2}\right)$. Consequently, if $0<|\lambda|<\lambda_{1}$, then for each $\omega \in \Omega_{1}$ we have $M(\omega) \in \mathcal{M}_{(E)}$, by Lemma 5.1. By assumption $(\mathrm{R} 1), \mathrm{p}\left(\Omega_{1}\right) \neq 0$, so (5.11) is proven.

\section{References}

[1] Attal, S., Joye, A.: Weak Coupling and Continuous Limits for Repeated Quantum Interactions Journ. Stat. Phys. (2007), DOI 10.1007/s10955-006-9085-z

[2] S. Attal, A. Joye, C.-A. Pillet (Eds.), Open Quantum Systems I-III, Lecture Notes in Mathematics, volumes 1880-1882, Springer Verlag, 2006.

[3] O. Bratteli, D.W. Robinson, Operator Algebras and Quantum Statistical Mechanics, volumes 1 and 2, Texts and Monographs in Physics, Springer Verlag, 1996.

[4] L. Bruneau, A. Joye, M. Merkli: Asymptotics of repeated interaction quantum systems, J. Func. Anal. 239, 310-344 (2006).

[5] L. Bruneau, A. Joye, M. Merkli: Infinite Products of Random Matrices and Repeated Interaction Dynamics, submitted, math.PR/0703675, mp-arc 07-67.

[6] L. Bruneau, A. Joye, M. Merkli: Random Repeated Interaction Quantum Systems, submitted, arXiv:0710.5908, mp-arc 07-262.

[7] V.Jaksic, C.-A.Pillet: Non-equilibrium steady states of finite quantum systems coupled to thermal reservoirs, Commun. Math. Phys. 226, 131-162 (2002).

[8] Kato, K., Perturbation Theory for Linear Operators. 2nd edition. Springer, Berlin, 1976. 
[9] D. Meschede, H. Walther, G. Müller: One-atom maser, Phys. Rev. Lett. 54, 551-554 (1993).

[10] Merkli, M., Mück, M., Sigal, I.M.: Instability of Equilibrium States for Coupled Heat Reservoirs at Different Temperatures, J. Funct. Anal. 243, 87-120 (2007).

[11] M. Weidinger, B.T.H. Varcoe, R. Heerlein, H. Walther: Trapping states in micromaser, Phys. Rev. Lett. 82, 3795-3798 (1999).

[12] T. Wellens, A. Buchleitner, B. Kümmerer, H. Maassen: Quantum state preparation via asymptotic completeness, Phys. Rev. Lett. 85, 3391-3364 (2000). 